\title{
sensors
}

ISSN 1424-8220

(C) 2002 by MDPI

http://www.mdpi.net/sensors

\section{Mediated Electron Transfer at Redox Active Monolayers . Part 2 : Analysis of the Chronoamperometric Response to a Potential Step Perturbation.}

\author{
Michael E.G. Lyons
}

Physical Electrochemistry Laboratory, Department of Chemistry, Nasr Institute of Advanced Materials Science, University of Dublin, Trinity College, Dublin 2, Ireland. Tel: 353-1-608 2051. Fax: 353-1671 2826. E-mail: melyons@tcd.ie.

Received: 2 July 2002 / Accepted: 6 August 2002 / Published: 17 August 2002

\begin{abstract}
A theoretical model describing the transport and kinetic processes involved in heterogeneous redox catalysis of solution phase reactants at electrode surfaces coated with redox active monolayers is presented. We describe theoretically the time dependent chronoamperometric response expected for a redox active monolayer in the absence of a substrate in solution, and subsequently extend the analysis to consider the reaction of a solution phase substrate mediated by surface immobilized redox groups. This is accomplished via a Laplace transform based solution of the Fick diffusion equation for the substrate transport to the monolayer surface coupled with the development of a suitable flux matching condition at the monolayer/solution interface. The latter procedure enables the development of an analytical expression for the transient current response in terms of well established mathematical special functions. We have shown that kinetic information may be readily extracted from the developed expressions for the current response as a function of time. In the present paper we assume that the reaction between mediator and substrate is of a simple outer sphere bimolecular type.
\end{abstract}

Keywords: Heterogeneous redox catalysis, Redox active monolayers, Chronoamperometry of redox active monolayers, Monolayer modified electrodes.

\section{Introduction}

The phenomenon of heterogeneous redox catalysis at electrode surfaces has been studied extensively over the last twenty years. Much attention has been focused on chemically modified electrodes, including, for example, electrodes modified with electroactive polymer films [1], adsorbed 
redox active dye molecules [2], conductive oxide surfaces [3] , and, more recently, metallic electrodes coated with adsorbed redox active monolayers which are generated via self assembly mechanisms [4].

Redox mediation is simple in concept. In this process surface immobilized sites may be activated electrochemically via application of a voltage to the support electrode surface. The latter sites may then oxidize or reduce other redox agents located in the solution phase adjacent to the immobilized layer, for which the direct oxidation or reduction at the electrode surface is inhibited, either because of intrinsically slow heterogeneous electron transfer kinetics, or because close approach of the soluble redox species to the electrode is prevented.

Mediated electron transfer of solution phase species at electrode surfaces containing immobilized redox species can be examined experimentally using a number of electrochemical techniques. The technique of rotating disc voltammetry is most often applied, since in principle, the processes of reactant transport in solution, and kinetic processes at the monolayer can be cleanly separated, by conducting voltammetric experiments over a range of rotation speeds. Previous theoretical work describing mediated redox catalysis at monolayers in the context of rotating disc voltammetry has been presented by Andrieux and Saveant [5], and Laviron [6], More recently the analysis has been extended to a time dependent technique such as cyclic voltammetry by Aoki and co-workers [7] and by Xie and Anson [8-10]. The latter analysis of the cyclic voltammetric response can be quite complex and difficult.

In the first paper of the present series, a general steady state analysis of mediated electron transfer at redox active monolayer surfaces was presented [11]. It is interesting to note that the technique of potential step chronoamperometry has not been used in any extensive manner to examine mediated electron transfer processes at redox active monolayers. This is surprising given that the technique is simple both in concept and in execution. A singular example extracted from the literature is an early paper published by Rocklin and Murray [12]. In this account the potential step technique was utilized and the concept validated for the reduction of dibromoalkanes at electrode surfaces chemically modified with thin immobilized metallo tetraphenyl porphyrins. In the present paper we present a theoretical analysis of potential step chronoamperometry applied to redox active monolayer films and indicate how the technique may be used to obtain transport and kinetic information from experiment.

In this paper we initially describe theoretically the chronoamperometric response expected for a redox active monolayer firstly in the absence of a substrate in solution, and subsequently extend the analysis to consider the reaction of a solution phase substrate mediated by surface immobilized redox groups. In the present paper we assume that the reaction between mediator and substrate is of a simple outer sphere bimolecular type. The more challenging problem where the possibility that distinct adduct between surface immobilized mediator and substrate is considered, will be presented in the succeeding paper.

\section{Chronoamperometric Response of Surface Immobilized Redox Groups in the Absence of Solution Phase Substrate}

We initially consider the transient current response obtained to a large amplitude potential step perturbation applied to a surface immobilized redox couple $A / B$. We let $\Gamma_{j}(j=A, B)$ represent the 
surface coverage (units: $\mathrm{mol} \mathrm{cm} \mathrm{cm}^{-2}$ ) of component $\mathrm{j}$ of the redox couple, and define the total surface coverage as $\Gamma_{\Sigma}=\Gamma_{A}+\Gamma_{B}$. We initially assume that the surface redox reaction is irreversible with a rate equation given by:

$$
-\frac{d \Gamma_{A}}{d t}=\frac{d \Gamma_{B}}{d t}=k_{E}^{\prime} \Gamma_{A}
$$

where the heterogeneous electrochemical rate constant (units: $\mathrm{s}^{-1}$ ) is of the Butler-Volmer type and is given by

$$
k_{E}^{\prime}=k^{0} \exp [\beta \xi]
$$

where $\mathrm{k}^{0}$ denotes the standard rate constant, $\beta$ is the symmetry factor and $\xi$ denotes a normalized potential given by $\xi=\frac{F}{R T}\left(E-E_{A / B}^{0}\right)$. The current response to the applied potential step is given by

$$
i(t)=n F A f_{\Sigma}(t)=n F A k_{E}^{\prime} \Gamma_{A}(t)
$$

where $f_{\Sigma}$ denotes the net flux (units: $m o l \mathrm{~cm}^{-2} \mathrm{~s}^{-1}$ ), $\mathrm{n}$ denotes the number of electrons transferred in the surface redox process, A denotes the geometric area of the electrode and $\mathrm{F}$ is the Faraday constant.

The variation of surface coverage with time is obtained via integration of eqn. 1 subject to the initial condition that at $t=0 \quad \Gamma_{A}=\Gamma_{\Sigma}$. Hence integration of eqn.1 immediately yields that

$$
\Gamma_{A}(t)=\Gamma_{\Sigma} \exp \left[-k_{E}^{\prime} t\right]
$$

and the surface redox transition obeys first order kinetics. Hence the current response is expected to follow a simple exponential decay (figure 1) and is given by

$$
i(t)=k_{E}^{\prime} Q \exp \left[-k_{E}^{\prime} t\right]
$$

where the charge $\mathrm{Q}$ is related to the surface coverage of electroactive groups via the expression $Q=n F A \Gamma_{\Sigma}$. This expression has been used by Finklea [13], Chidsey [14], Miller and co-workers [15] and by Forster and Faulkner [16] to examine the dynamics of self assembled monolayer systems.
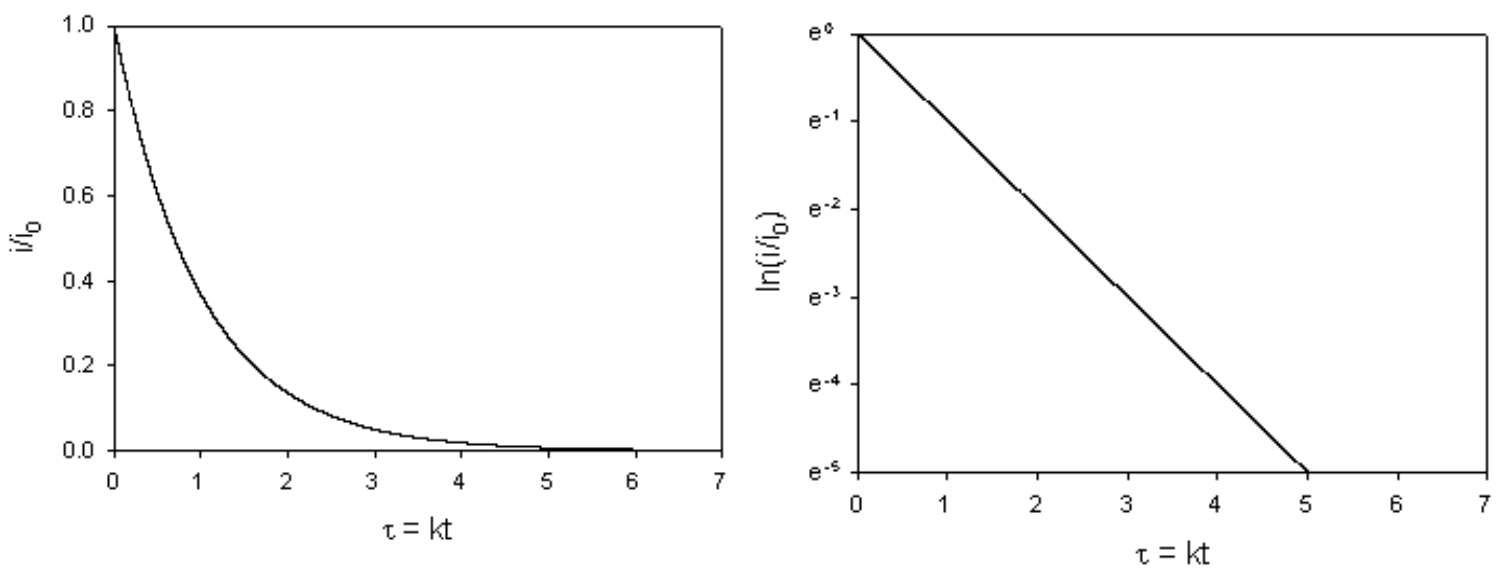

Figure 1. Plot of normalized current versus normalized time corresponding to the chronoamperometric response profile of a redox active monolayer. The linear semilogarithmic plot is characteristic of this system. 
We can readily extend the analysis to consider a quasi-reversible surface reaction. In this case the rate equation for the surface redox reaction is given by

$$
-\frac{d \Gamma_{A}}{d t}=k_{E}^{\prime} \Gamma_{A}-k_{-E}^{\prime} \Gamma_{B}
$$

where the heterogeneous rate constant for the reverse step is given by $k_{-E}^{\prime}=k^{0} \exp [-(1-\beta) \xi]$. We can integrate eqn.6 using the initial condition $t=0 \quad \Gamma_{A}=\Gamma_{\Sigma} \quad \Gamma_{B}=0$ to obtain

$$
\Gamma_{A}(t)=\Gamma_{\Sigma}\left\{\frac{1+\left(k_{E}^{\prime} / k_{-E}^{\prime}\right) \exp \left[-k_{\Sigma} t\right]}{1+\left(k_{E}^{\prime} / k_{-E}^{\prime}\right)}\right\}
$$

where we note that $k_{\Sigma}^{\prime}=k_{E}^{\prime}+k_{-E}^{\prime}$. At any time $\mathrm{t}$ we also note that $\Gamma_{A}(t)+\Gamma_{B}(t)=\Gamma_{\Sigma}$ and so we also obtain that

$$
\Gamma_{B}(t)=\Gamma_{\Sigma}\left\{\frac{\left(k_{E}^{\prime} / k_{-E}^{\prime}\right)\left(1-\exp \left[-k_{\Sigma} t\right]\right)}{1+\left(k_{E}^{\prime} / k_{-E}^{\prime}\right)}\right\}
$$

The net current response is therefore given by

$$
i(t)=n F A\left\{k_{E}^{\prime} \Gamma_{A}(t)+k_{-E}^{\prime} \Gamma_{B}(t)\right\}
$$

From eqn.7, eqn.8 and eqn.9 we immediately obtain

$$
i(t)=n F A k_{E}^{\prime} \Gamma_{\Sigma} \exp \left[-k_{\Sigma} t\right]
$$

Again a simple exponential decay in current with time is predicted. A plot of $\ln \mathrm{i}$ versus $\mathrm{t}$ is linear with intercept given by $n F A k_{E}^{\prime} \Gamma_{\Sigma}$ and a slope given by $-k_{\Sigma}^{\prime}$. Hence the surface redox kinetics can be completely resolved using the potential step technique.

We expect that both the slope and the intercept of the first order kinetic plot be potential dependent. Finklea and Hanshew [17] have indicated that examination of the heterogeneous electrochemical rate constants provides a stringent test for structural order within redox active monolayer films. If the monolayer is ordered then little spread in the electrochemical rate constant should be observed. Finklea and Hanshew [17] have shown via simulation techniques, that if a spread in heterogeneous rate constants is assumed, then the chronoamperometric plot of $\ln \mathrm{i}$ versus $\mathrm{t}$ has an apparent slope that decreases in magnitude as time increases. Hence the current flow at short times is dominated by the faster rate constants while current flow at longer times is dominated by the slower rate constants. Hence a bi-phasic, 'dog leg' type of profile should be observed. The useful feature of chronoamperometric plots is that if the latter exhibit good linearity then theories of long range electron transfer can be carefully tested. This has been done in some detail in recent papers [18-21].

It is also relatively easy to include interaction effects between the immobilized redox groups in the analysis. We can follow Xie and Anson [8-10] and introduce an interaction parameter g into the Butler-Volmer expression for the heterogeneous rate constants. Hence we can write that

$$
\begin{aligned}
& k_{E}^{\prime}=k^{0} \exp [\beta g \xi] \\
& k_{-E}^{\prime}=k^{0} \exp [-(1-\beta) g \xi]
\end{aligned}
$$

where the interaction parameter $g$ accounts for mutual interactions between the immobilized redox sites on the electrode surface. When $g=1$ the interaction is negligible, and we have the ideal situation. 
When $\mathrm{g}$ is negative repulsive interactions between the redox groups pertain and when $\mathrm{g}$ is positive attractive interactions operate. If the surface redox reaction is Nernstian then the pertinent expression is given by [22] :

$$
E=E^{0}+\frac{R T}{g n F} \ln \left\{\frac{\Gamma_{B}}{\Gamma_{A}}\right\}
$$

Interaction effects have been discussed in detail by Brown and Anson [22], Albery and Colby [23], Laviron [24] and by Tokuda and co-workers [25,26]. A recent summary of work in this area has been provided by Lyons [1].

\section{Chronoamperometric Response of Surface Immobilized Redox Groups in the Presence of Solution Phase Substrate}

We now address the main issue of the present paper, that of mediated electron transfer between a solution phase reactant and a redox site immobilized at a monolayer coverage on a support electrode surface. We consider the following reaction scheme:

$$
\begin{aligned}
& A \stackrel{\mp n e^{-}}{\longrightarrow} B \\
& S+B \rightarrow P+A
\end{aligned}
$$

In the following analysis we let $\mathrm{A}$ and $\mathrm{B}$ represent the reduced and oxidized forms of the surface immobilized redox couple, and $\mathrm{S}$ and $\mathrm{P}$ the substrate (reactant species) and product respectively. We shall assume that the cross exchange reaction between the substrate species $\mathrm{S}$ and the redox mediator species $\mathrm{B}$ is irreversible, and we will assume that the kinetics of the A/B surface redox transformation is facile and is described by the Nernst equation. We will also briefly comment on the more complex situation in which the mediator generation kinetics are irreversible and described by a kinetic expression of the Butler-Volmer form. We assume that the net current response will appear as the sum of two contributions $i=i_{\text {surf }}+i_{\text {cat }}$ where $i_{\text {surf }}$ is the current obtained in the absence of solution phase substrate and $i_{\text {cat }}$ denotes the catalytic current contribution. Clearly the $i_{\text {surf }}$ term will be given by either eqn. 5 or eqn.10. In the following we focus attention totally on the catalytic term.

\section{Irreversible Mediated Electron Transfer with Nernstian Mediator Generation}

We first consider the simple situation where immobilized mediator generation is Nernstian and hence kinetically facile and the mediated bimolecular electron transfer reaction between the solution phase substrate and the immobilized mediator species is irreversible.

The net flux is given by

$$
f_{\Sigma}=\frac{i}{n F A}=k_{H} \Gamma_{B} s_{0}
$$

where $\mathrm{k}_{\mathrm{H}}$ represents the bimolecular cross exchange rate constant and $\mathrm{s}_{0}$ denotes the substrate concentration at the monolayer/solution interface. If the heterogeneous electron transfer process is 
Nernstian then the surface coverage of redox mediator species will depend on the normalized potential according to

$$
\begin{aligned}
\Gamma_{B} & =\frac{\Gamma_{\Sigma}}{1+\exp [-\xi]} \\
\Gamma_{A} & =\frac{\Gamma_{\Sigma}}{1+\exp [\xi]}
\end{aligned}
$$

If the magnitude of the potential step perturbation is large then we immediately note that $\Gamma_{B}=\Gamma_{\Sigma}$. The main challenge is to determine the interfacial concentration of substrate $\mathrm{s}_{0}$. This is accomplished by solving the time dependent Fick diffusion equation for substrate transport to the monolayer surface

$$
\frac{\partial s}{\partial t}=D_{S} \frac{\partial^{2} s}{\partial x^{2}}
$$

subject to the following initial and boundary conditions

$$
s(x, 0)=s^{\infty} \quad s(\infty, t)=s^{\infty}
$$

and the following flux matching condition at the electrode surface

$$
\frac{d \Gamma_{B}}{d t}=D_{S}\left(\frac{d s}{d x}\right)_{0}=k_{H} \Gamma_{B} s_{0}
$$

In eqn.17 $\mathrm{D}_{\mathrm{S}}$ denotes the diffusion coefficient of the substrate species in the solution, and finally, $\mathrm{s}^{\infty}$ represents the bulk concentration of substrate.

We introduce the following normalized variables

$$
u=\frac{s}{s^{\infty}} \quad \chi=\frac{x}{\delta} \quad \tau=\frac{t}{t^{*}}
$$

where $t^{*}$ denotes the typical experimental timescale. The diffusion layer thickness $\delta$ is time dependent, and is given by $\delta=\sqrt{D_{S} t^{*}}$. Hence in normalized variables the Fick diffusion equation becomes

$$
\frac{\partial u}{\partial \tau}=\frac{\partial^{2} u}{\partial \chi^{2}}
$$

and the initial and boundary conditions transform to

$$
u(\chi, 0)=1 \quad u(\infty, \tau)=1 \quad\left(\frac{d u}{d \chi}\right)_{0}=\gamma u_{0}
$$

where we introduce the reaction/diffusion parameter $\gamma$ as

$$
\gamma=\frac{k_{H} \Gamma_{B} \delta}{D_{S}}
$$

and we note that $u_{0}=\frac{s_{0}}{s^{\infty}}$.

The method of Laplace transformation may be utilized to solve the problem. In Laplace space the differential equation presented in eqn.19 transforms to 


$$
\frac{d^{2} \bar{u}}{d \chi^{2}}=1+p \bar{u}
$$

where $\bar{u}$ is the normalized substrate concentration in Laplace space and $\mathrm{p}$ denotes the Laplace parameter. The boundary conditions in Laplace space adopt the following representation

$$
\bar{u}(\infty, p)=\frac{1}{p} \quad\left(\frac{d \bar{u}}{d \chi}\right)_{0}=\gamma \bar{u}_{0}
$$

The normalized flux is given by the expression

$$
\Psi=\frac{f_{\Sigma} \delta}{D_{S} s^{\infty}}=\left(\frac{d u}{d \chi}\right)_{0}
$$

We can readily show that a general solution to eqn. 22 is given by

$$
\bar{u}(\chi, p)=\frac{1}{p}+A(p) \exp [\sqrt{p} \chi]+B(p) \exp [-\sqrt{p} \chi]
$$

Since the concentration $\bar{u}$ must be finite as $\chi \rightarrow \infty$ then clearly $\mathrm{A}=0$. Differentiation of eqn. 25 with respect to $\chi$ and using the second relation in eqn.23 we obtain that $B=-\gamma p^{-1 / 2} \bar{u}_{0}$. Noting that $\bar{u}(\chi, p)=\frac{1}{p}-\frac{\gamma}{\sqrt{p}} \bar{u}_{0}$, and the requirement that at $\chi=0 \bar{u}=\bar{u}_{0}$, we can readily show that $\bar{u}_{0}=\frac{1}{p}\left\{\frac{\sqrt{p}}{\sqrt{p}+\gamma}\right\}$. Consequently we note that $B=-\frac{\gamma}{p(\sqrt{p}+\gamma)}$.

Hence in Laplace space the concentration profile of substrate is given by

$$
\bar{u}(\chi, p)=\frac{1}{p}-\frac{\gamma \exp [-\sqrt{p} \chi]}{p(\sqrt{p}+\gamma)}
$$

The concentration profile of substrate is obtained from eqn.26 via inverse Laplace Transformation. Hence

$$
u(\chi, \tau)=1-\gamma\left\{L-1\left\{\frac{\exp [-\sqrt{p} \chi]}{p(\sqrt{p}+\gamma)}\right\}\right\}
$$

We note that

$$
L^{-1}\left\{\frac{\exp [-\alpha \sqrt{p}]}{p(\sqrt{p}+\beta)}\right\}=\frac{1}{\beta} \operatorname{erfc}\left[\frac{\alpha}{2 \sqrt{\tau}}\right]-\frac{1}{\beta} \exp \left[\alpha \beta+\beta^{2} \tau\right] \operatorname{erfc}\left[\beta \sqrt{\tau}+\frac{\alpha}{2 \sqrt{\tau}}\right]
$$

Utilizing the latter identity we immediately note that

$$
u(\chi, \tau)=1-\left\{\operatorname{erfc}\left[\frac{\chi}{2 \sqrt{\tau}}\right]-\exp \left[\gamma \chi+\gamma^{2} \tau\right] \operatorname{erfc}\left[\gamma \sqrt{\tau}+\frac{\chi}{2 \sqrt{\tau}}\right]\right\}
$$

We can also readily obtain an expression for the normalized flux. We note that 


$$
\bar{\Psi}(p)=\left(\frac{d \bar{u}}{d \chi}\right)_{0}=\frac{\gamma}{\sqrt{p}(\sqrt{p}+\gamma)}
$$

Hence in real space the normalized flux is given by

$$
\begin{aligned}
\Psi(\tau) & =\gamma\left\{L-1\left\{\frac{1}{\sqrt{p}(\sqrt{p}+\gamma)}\right\}\right\} \\
& =\gamma \exp \left[\gamma^{2} \tau\right] \operatorname{erfc}[\gamma \sqrt{\tau}]
\end{aligned}
$$

It is interesting to make the observation that eqn.31 is of the same form as the established relationship for slow charge transfer at an unmodified electrode surface [27]. In this case the rate constant $\mathrm{k}_{\mathrm{H}}$ for the cross exchange reaction between the substrate and the immobilized mediator species is to be identified with the potential dependent heterogeneous rate constant $\mathrm{k}$ ' for electron transfer at the bare electrode. Indeed we can make the identification as $k^{\prime}=k_{H} \Gamma_{B}=\frac{k_{H} \Gamma_{\Sigma}}{1+\exp [-\xi]}$. We note however that the heterogeneous electron transfer rate constant k' increases exponentially with increasing potential $\xi$ according to the Butler-Volmer law, unless rate determining diffusive transport becomes rate limiting, whereas the composite term $k_{H} \Gamma_{B} \rightarrow k_{H} \Gamma_{\Sigma}$ as $\xi \rightarrow \infty$. We can readily show that the current response as a function of time is given by

$$
i(t)=n F A k_{H} \Gamma_{B} s^{\infty} \exp \left[\frac{k_{H}{ }^{2} \Gamma_{B}{ }^{2} t}{D_{S}}\right] \operatorname{erfc}\left[\frac{k_{H} \Gamma_{B} \sqrt{t}}{\sqrt{D_{S}}}\right]
$$

where the surface coverage of immobilized mediator species is given by

$$
\Gamma_{B}=\frac{\Gamma_{\Sigma}}{1+\exp \left[-\frac{n F\left(E-E^{0}{ }_{A / B}\right)}{R T}\right]}
$$

This expression will be valid when the heterogeneous kinetics involving mediator generation are rapid and described by the Nernst equation.

We return to eqn.31 and compare the latter expression for the normalized chronoamperometric current transient with that predicted for a simple diffusion controlled reaction which is given by the Cottrell equation [28]. In normalized form the Cottrell equation is given by

$$
\Psi_{C}(\tau)=\frac{1}{\sqrt{\pi \tau}}
$$

Consequently we examine the ratio of the catalytic and the Cottrell expressions to obtain

$$
\frac{\Psi(\tau)}{\Psi_{C}(\tau)}=\sqrt{\pi} \gamma \sqrt{\tau} \exp \left[\gamma^{2} \tau\right] \operatorname{erfc}[\gamma \sqrt{\tau}]
$$

We set $\lambda=\gamma^{2} \tau$ and obtain the following well established result 


$$
\frac{\Psi(\tau)}{\Psi_{C}(\tau)}=F(\lambda)=\sqrt{\pi \lambda} \exp [\lambda] \operatorname{erfc}[\sqrt{\lambda}]
$$

The properties of the $F(\lambda)$ function have been described in detail in the useful monograph authored by Spanier and Oldham [29]. Two limiting cases can be derived.

Firstly, when $\lambda$ is large corresponding to the limit of long times

$$
F(\lambda) \cong 1-\frac{1}{2 \lambda}+\frac{3}{4 \lambda^{2}}-\cdots+\frac{(2 j-1) ! !}{(-2 \lambda)^{j}}+\cdots \cong 1
$$

Hence for large $\lambda$ or large $\tau$ we note that

$$
\Psi(\tau) \cong \Psi_{C}(\tau)=\frac{1}{\sqrt{\pi \tau}}
$$

and the chronoamperometric response corresponding to mediated electrocatalysis is under simple diffusion control and is well described by the Cottrell equation.

Secondly, when $\lambda$ is small corresponding to short times we note that

$$
\exp [\lambda] \operatorname{erfc}[\sqrt{\lambda}] \cong 1-2 \sqrt{\frac{\lambda}{\pi}}
$$

and the normalized chronoamperometric response takes the form:

$$
\Psi(\tau) \cong \sqrt{\frac{\lambda}{\tau}}\left\{1-2 \sqrt{\frac{\lambda}{\pi}}\right\}=\gamma-\frac{2 \gamma^{2}}{\pi^{1 / 2}} \sqrt{\tau}
$$

Hence in the limit of short times a plot of $\Psi(\tau)$ versus $\tau^{1 / 2}$ is linear with a negative slope given by $\frac{2 \gamma^{2}}{\sqrt{\pi}}$ and an intercept given by the reaction/diffusion parameter $\gamma$. Hence analysis of the chronoamperometric data at short times can produce kinetic information.

Spanier and Oldham [29] have noted that for any value of the parameter $\lambda$ the following approximation holds

$$
F(\lambda)=\sqrt{\pi \lambda} \exp [\lambda] \operatorname{erfc}[\sqrt{\lambda}] \cong \frac{2}{1+\sqrt{1+\frac{2}{\lambda}\left\{1-\frac{\left(1-\frac{2}{\pi}\right)}{\exp \left[\sqrt{\frac{5 \lambda}{7}}\right]}\right.}}
$$

This approximation is very useful for numerical work and exploits the inequality

$$
\frac{2}{1+\sqrt{1+\frac{2}{\lambda}}}<F(\lambda) \leq \frac{2}{1+\sqrt{1+\frac{4}{\pi \lambda}}}
$$

Hence we conclude that to a good approximation, the normalized chronoamperometric transient response profile is well described by 


$$
\Psi(\tau) \cong \frac{2}{\sqrt{\pi \tau}}\left\{1+\left\{1+\frac{2}{\gamma^{2} \tau}\left(1-\frac{(1-2 / \pi)}{\exp \left[\sqrt{5 \gamma^{2} \tau / 7}\right]}\right)\right\}^{1 / 2}\right\}^{-1}
$$

The latter expression is presented in figures 2-4 both as a function of the reaction diffusion parameter $\gamma$ and the normalized potential $\xi$. We recall that $\gamma=\frac{k_{H} \Gamma_{B} \delta}{D_{S}}$ and $\Gamma_{B}=\frac{\Gamma_{\Sigma}}{1+\exp \left[-\frac{n F\left(E-E^{0}{ }_{A / B}\right)}{R T}\right]}$ and so the $\gamma$ parameter is potential dependent given by $\gamma=\frac{k_{H} \Gamma_{\Sigma} \delta}{D_{S}}\left\{\frac{1}{1+\exp [-\xi]}\right\}$ where we recall that the normalized potential is given by $\xi=\frac{F}{R T}\left(E-E_{A / B}^{0}\right)$. In the computations presented in figures 2-4 the $\gamma$ values quoted correspond to the quantity $\frac{k_{H} \Gamma_{\Sigma} \delta}{D_{S}}$.

In figure 2-4 we present the normalized chronoamperometric transient response calculated using the SigmaPlot software package (SigmaPlot 2001, SPSS Science) from eqn.43. In all figures the $\Psi$ versus $\tau$ profiles are produced for various values of the normalized potential $\xi$. The latter curves are then presented in Cottrell format $\left(\Psi\right.$ versus $\tau^{-1 / 2}$ ) and as plots of $\Psi$ versus $\tau^{1 / 2}$. In figure 2 the analysis is presented for the reaction/diffusion parameter $\gamma=0.1$. In figure 3 the analysis is repeated for $\gamma=1$ and in figure 4 the situation corresponding to $\gamma=10$ is presented. We recall that the $\gamma$ parameter quantifies the balance between heterogeneous kinetics involving the immobilized mediator and substrate diffusion. Hence $\gamma=\frac{k_{H} \Gamma_{B} \delta}{D_{S}}=\frac{f_{K}}{f_{D}}$ where $\mathrm{f}_{\mathrm{K}}$ denotes the kinetic flux arising from reaction between immobilized mediator species $B$ and substrate $S$ and $f_{D}$ represents the flux due to diffusive material transport of substrate to the site of reaction on the surface of the redox monolayer. When $\gamma$ is small (as in figure 2), substrate diffusion is more rapid than heterogeneous kinetics. Hence the data when displayed in the Cottrell format will be non linear over an extensive period of the experimental timescale. Linear Cottrell behaviour will only be observed at the longest times. In contrast a plot of $\Psi$ versus $\tau^{1 / 2}$ will exhibit good linearity over an extended timescale. This is in accord with the approximate expression presented in eqn.40 which holds when the parameter $\lambda=\gamma^{2} \tau$ is small. If $\gamma$ is small enough then $\lambda$ will be small over an extended period of the normalized time $\tau$. A well defined intercept is obtained on the $\Psi$ axis of the plot, the magnitude of which will depend on the value of the normalized potential $\xi$, since we recall that $\gamma=\frac{k_{H} \Gamma_{\Sigma} \delta}{D_{S}}\left\{\frac{1}{1+\exp [-\xi]}\right\}$.

In figure 3 we present an analysis of eqn.43 when $\gamma=1$. Here the substrate diffusion flux and the flux arising from the bimolecular heterogeneous mediation kinetics are in balance. We note that a linear region is more marked in the Cottrell plot whereas a direct plot of $\Psi$ versus $\tau^{1 / 2}$ exhibits linearity only at short times. This behaviour is even more apparent in the computations presented in figure 4 where $\gamma=10$. Here the diffusive flux is much less than the heterogeneous kinetic flux and rate control 
via diffusive transport of substrate pertains. Cottrell plots are linear over an extended time domain and the plots of $\Psi$ versus $\tau^{1 / 2}$ are non-linear except at very short times.

We note that the computed curves presented in figures 2-4 are similar in shape to experimentally determined chronoamperometric profiles recorded by Rocklin and Murray [12]. In this work a Pt surface was modified with an immobilized cobalt(II) tetra(p-aminophenyl) porphyrin monolayer, which exhibited redox activity according to the reaction $\mathrm{Co}^{I I}\left(\mathrm{NH}_{2}\right)_{4} \mathrm{TPP}+e^{-} \rightarrow \mathrm{Co}^{I}\left(\mathrm{NH}_{2}\right)_{4} \mathrm{TPP}$. Reference is made especially to figure 11 of the latter paper, in which a Cotrell analysis of the experimentally determined chronoamperometric transient for the reduction of the substrate species $\mathrm{PhCHBrCHBrPh}$ at a Pt electrode modified with cobalt(II) tetra(p-aminophenyl) porphyrin monolayer, exhibited linear behaviour only at long times. A marked deviation from linearity corresponding to rate determining heterogeneous kinetics involving the immobilized $\mathrm{Co}^{\mathrm{I}}$ metallo-porphyrin species and the dihaloaromatic substrate species, was observed over much of the experimental time-scale.
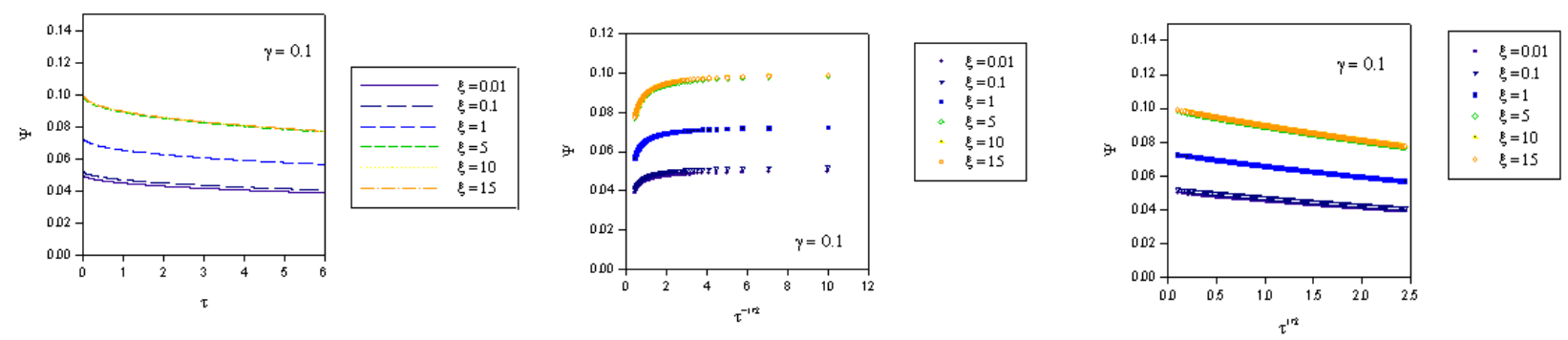

Figure 2. Plots of normalized chronoamperometric response in various formats derived from eqn.43 for $\gamma=0.1$.
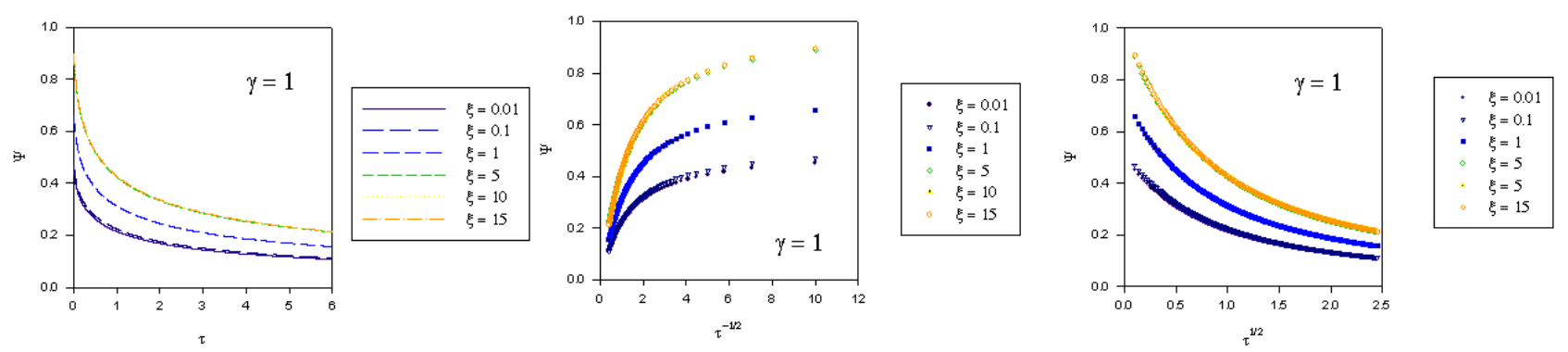

Figure 3. Plots of normalized chronoamperometric response in various formats derived from eqn. 43 for $\gamma=1$.
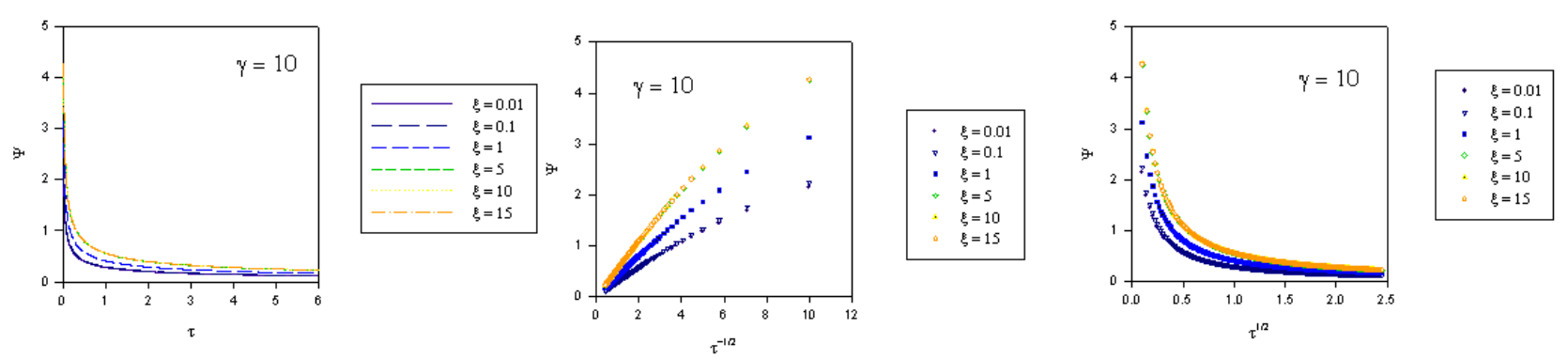

Figure 4. Plots of normalized chronoamperometric response in various formats derived from eqn.43 for $\gamma=10$. 


\section{Irreversible Mediated Electron Transfer Coupled with Irreversible Heterogeneous Electron Transfer Kinetics}

Here we examine the more complicated situation of mediated electron transfer at a monolayer film assuming that the bimolecular cross exchange reaction is irreversible, and that mediator generation is irreversible and described via Butler-Volmer kinetics.

In this case the net flux is given by

$$
f_{\Sigma}=\frac{i}{n F A}=D_{S}\left(\frac{d s}{d x}\right)_{0}=k_{E}^{\prime} \Gamma_{A}+k_{H} \Gamma_{B} s_{0}
$$

and so the flux consists of two terms : that due to the surface reaction involving mediator generation, and that arising from the mediation cross exchange reaction involving B and S. Again to obtain an expression for the interfacial concentration of substrate in eqn.44, the time dependent Fick diffusion equation presented in eqn.15 must be solved subject to the initial and boundary conditions presented in eqn.16. However the flux balance at the interface is now expressed via eqn.44. Again we adopt the strategy of using normalized variables to make the equations dimensionless and utilize the definitions presented in eqn.18. We can readily show that the flux matching condition for the irreversible generation of a surface bound redox mediator species presented in eqn.34 can be expressed as

$$
\left(\frac{d u}{d \chi}\right)_{0}=\zeta(1-\theta)+\gamma^{\prime} u_{0} \theta
$$

where we note that $\theta=\frac{\Gamma_{B}}{\Gamma_{\Sigma}}, \gamma^{\prime}$ is given by a modified form of eqn.21 and is represented as $\gamma^{\prime}=\frac{k_{H} \Gamma_{\Sigma} \delta}{D_{S}}$, and the heterogeneous rate constants are of the Butler-Volmer form $k_{E}^{\prime}=k^{0} \exp [\beta \xi]$.We have also introduced a further competition parameter $\zeta$ which relates the flux associated with mediator generation with the substrate diffusion flux. This parameter is given by $\zeta=\frac{k_{E}^{\prime} \Gamma_{\Sigma} \delta}{D_{S} s^{\infty}}$

A problem immediately arises if we want to apply the method of Laplace Transformation to solve the problem as we have done for the situation where surface mediator generation is Nernstian. We note from eqn.45 that in general both the normalized concentration of the substrate at the monolayer/solution interface $\mathrm{u}_{0}$ and the fractional surface coverage $\theta$ are both time dependent. Indeed we can readily show, using an analysis similar to that leading up to eqn.10, that

$$
\theta(\tau)=1-\exp [-\kappa \exp [\beta \xi] \tau]
$$

where the symmetry factor $\beta$ and the normalized potential $\xi$ have been invoked previously in eqn.2, and we have introduced the parameter $\kappa=\frac{k^{0} \delta^{2}}{D_{S}}$ and the normalized time $\tau=\frac{D_{S} t}{\delta^{2}}$. The variation of normalized surface coverage of mediator species with time, computed from eqn.46 is presented in figure 5 , for various values of the competition parameter $\kappa$ and the normalized potential $\xi$. It is clear from the latter computation that $\theta \rightarrow 1$ rather rapidly when either $\kappa$ increases or $\xi$ increases. 
It will be difficult to evaluate the Laplace transform of the bimolecular factor $\theta \mathrm{u}_{0}$ in eqn. 45 since in general the Laplace transformation operator is not distributive. However for irreversible kinetics the normalized potential $\xi$ applied to the electrode surface will usually be large (a large amplitude potential step will usually be applied) and consequentially the exponential term in $\beta \xi$ in eqn.46 will be very large. This situation will pertain, as illustrated in figure 5, provided that the kinetics of mediator generation within the monolayer is not very kinetically sluggish making the competition parameter $\kappa$ very much less than unity. Hence to a very good approximation we can assume that $\exp [-\kappa \exp [\beta \xi] \tau] \rightarrow 0$ under irreversible conditions where mediator generation is hard driven and so the normalized surface coverage of electrogenerated mediator species $\theta \cong 1$ and the quantity $1-\theta \cong 0$. Hence for irreversible conditions the generalized flux matching condition outlined in eqn.35 reduces to $\left(\frac{d u}{d \chi}\right)_{0} \cong \gamma u_{0}$ which is the same as that used in eqn.20 when Nernstian mediator generation was discussed. We can use the expression for $\mathrm{u}_{0}$ developed in our previous analysis and hence we recall from eqn.30 that $\bar{\Psi}(p)=\left(\frac{d \bar{u}}{d \chi}\right)_{0}=\frac{\gamma}{\sqrt{p}(\sqrt{p}+\gamma)}$, which on application of the inverse Laplace Transformation results in the following expression for the normalized current response:

$$
\Psi(\tau)=\gamma^{\prime} \exp \left[\gamma^{\prime 2} \tau\right] \operatorname{erfc}\left[\gamma^{\prime} \sqrt{\tau}\right]
$$

This is similar in form to the result outlined in eqn. 31 with the proviso that the reaction/diffusion parameter $\gamma^{\prime}$ is potential independent in that it reflects the total surface concentration of mediator species $\Gamma_{\Sigma}$ rather than the surface concentration of active mediator species $\Gamma_{\mathrm{B}}$ as is the case in eqn.31.
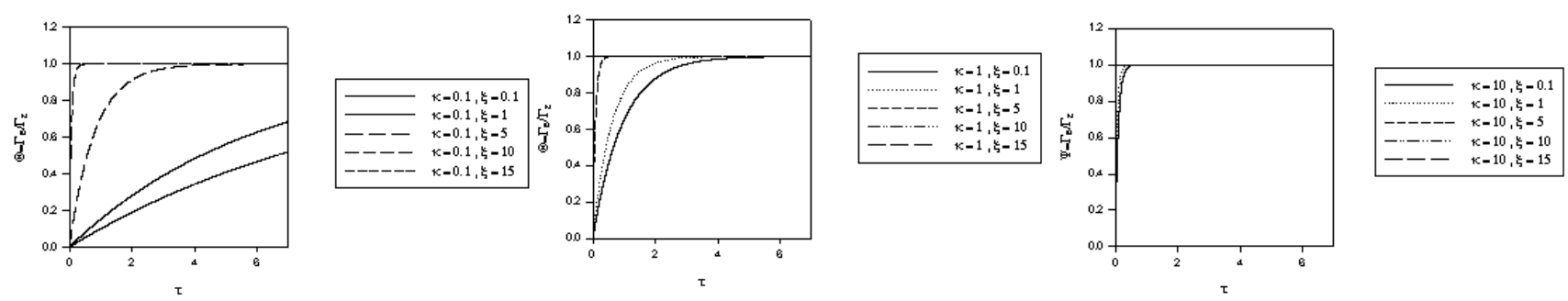

Figure 5. Variation in the normalized surface coverage of mediator species B with time for various values of $\kappa$ and normalized potential $\xi$.

\section{Discussion}

In the present communication we have presented a theoretical analysis describing the chronoamperometric response arising from the mediated electron transfer of a solution phase substrate at a redox active monolayer. Potential step chronoamperometry is one of the most simple transient electrochemical experiments to perform, and requires only the most basic electrochemical equipment . Consequently, we have found it surprising that this elementary technique has not been applied extensively to probe the kinetics at redox active monolayers. Most studies have utilized rotating disc voltammetry or cyclic voltammetry instead. Solution of the Fick diffusion equation for the substrate transport to the monolayer surface coupled with the development of a suitable flux matching condition 
at the monolayer/solution interface enabled the development of an analytical expression for the transient current response in terms of well established mathematical special functions. We have shown that kinetic information may be readily extracted from the developed expressions for the current response as a function of time.

It is important to note that our theory has assumed that a simple bimolecular reaction event occurs between the redox active surface immobilized mediator and the solution phase substrate and so the electron transfer event is of an outer sphere nature. This assumption may not be of general validity. For instance, as noted earlier, Rocklin and Murray [12] compared the electrocatalytic rates for different halo aliphatic and haloaromatic substrates such as $\mathrm{PhCHBrCH}_{2} \mathrm{Br}, \mathrm{PhCHBrCHBrPh}$ and $\mathrm{CH}_{2} \mathrm{BrCHBrCH}_{3}$ and the immobilized $\mathrm{Co}^{\mathrm{I}}$ metallo-porphyrin mediator. From this work they concluded that the electron transfer mediation process involved a specific interaction between substrate and metalloporphyrin, rather than being a simple bimolecular outer sphere event. It was proposed that the mediation reaction must involve the formation of an adduct species. However the authors [12] did not speculate on the nature of the substrate/binding mechanism or the mechanistic details of the electron transfer process. Bearing this observation in mind, we need to further develop the theoretical analysis of mediated electron transfer at redox active monolayers, to take into consideration a more complex kinetic interaction between mediator and substrate, involving binding and subsequent adduct formation. We will pursue this avenue of approach in the succeeding paper of the current series.

\section{References}

1. Lyons, M.E.G. Transport and kinetics in electroactive polymers. Adv. Chem. Phys. 1996, 94, 297624.

2. (a) Gorton, L. Chemically modified electrodes for the electrocatalytic oxidation of nicotinamide coenzymes. J. Chem. Soc., Faraday Trans. I 1986, 82, 1245-1258. (b) Gorton, L.; Torstensson, A.; Jaegfeldt, H.; Johansson, G. Electrocatalytic oxidation of reduced nicotinamide coenzymes by graphite electrodes modified with an adsorbed phenoxazinium salt, medola blue. J. Electroanal. Chem. 1984, 161, 103-120.

3. (a) Lyons, M.E.G.; Fitzgerald, C.A.; Smyth, M.R. Glucose oxidation at ruthenium dioxide based electrodes. Analyst 1994, 119, 855-861. (b) Lyons, M.E.G.; Lyons, C.H.; Michas, A.; Bartlett, P.N. Heterogeneous redox catalysis at hydrated oxide layers. J. Electroanal. Chem. 1993, 351, 245-258.

4. The electrochemistry of alkanethiol self assembled monolayers has been the subject of huge research activity in recent years. The following citations are representative of recent publications of interest in electroanalytical chemistry. (a) Gooding, J.J.; Hibbert, D.B. The application of alkanethiol self assembled monolayers to enzyme electrodes. Trends in Analytical Chemistry 1999, 18, 525- 533. (b) Gooding, J.J.; Erokhin, P.; Losic, D.; Yang, W.; Policarpio, V.; Liu, J.; Ho, F.M.; Situmorang, M.; Hibbert, D.B.; Shapter, J.G. Parameters important in fabricating enzyme electrodes using self assembled monolayers of alkanethiols. Analytical Sciences 2001, 17, 3-9. (c) Gooding, J.J.; Erokhin, P.; Hibbert, D.B. Parameters important in tuning the response of monolayer enzyme electrodes fabricated using self-assembled monolayers of alkanethiols. Biosensors and Bioelectronics. 2000, 15, 229-239. (d) Alleman, K.S.; Weber, K.; Creager, S.E. Electrochemical 
rectification at a monolayer modified electrode. J. Phys. Chem. 1996, 100, 17050-17058. (e) Retna Raj, C.; Ohsaka, T. Electrocatalytic sensing of NADH at an in situ functionalized self assembled monolayer on a gold electrode. Electrochemistry Communications 2001, 3, 633-638.

5. Andrieux, C.P.; Saveant, J.M. Heterogeneous versus homogeneous catalysis of electrochemical reactions. J. Electroanal. Chem. 1978, 93, 163-168.

6. Laviron, E. Electron transfer mediated by redox polymer electrodes. Theory for rotating disc voltammetry with transfer at the film/solution interface and reversible electrode coating reaction. $J$. Electroanal. Chem. 1982, 131, 61-75.

7. Aoki, K.; Tokuda, K.; Matsuda, H. Linear sweep and cyclic voltammetry for electrocatalysis at modified electrodes with very thin films. J. Electroanal. Chem. 1986, 199, 69-79.

8. Xie, Y.; Anson, F.C. Analysis of the cyclic voltammetric responses exhibited by electrodes modified with monolayers of catalysts in the absence and presence of substrates. J. Electroanal. Chem. 1995, 384, 145-153.

9. Xie, Y.; Anson, F.C. Calculation of cyclic voltammetric responses for the reduction of substrates by an inner sphere mechanism at electrode surfaces with single monolayers of catalysts. $J$. Electroanal. Chem. 1995, 396, 441-449.

10. Xie, Y.; Anson, F.C. Calculation of cyclic voltammetric responses for the catalytic reduction of substrates via intramolecular electron transfer within a catalyst/substrate complex formed on electrode surfaces. J. Electroanal. Chem. 1996, 404, 209-213.

11. Lyons, M.E.G.; Mediated electron transfer at redox active monolayers. Sensors. 2001, 1, 215-228.

12. Rocklin, R.D.; Murray, R.W. Kinetics of electrocatalysis of dibromoalkyl reductions using electrodes with covalently immobilized metallotetraphenylporphyrins. J. Phys. Chem. 1981, 85, 2104-2112.

13. (a) Finklea, H.O.; Liu, L.; Ravenscroft, M.S.; Punturi, S. Multiple electron tunneling paths across self-assembled monolayers of alkanethiols with attached ruthenium(II/III) redox centers. J. Phys. Chem. 1996, 100, 18852-18858. (b) Finklea, H.O. Consequences of a potential dependent transfer coefficient in ac voltammetry and in coupled electron-proton transfer for attached redox couples. $J$. Electroanal. Chem. 2001, 495, 79-86. (c) Finklea, H.O.; Yoon, K.; Chamberlain, E.; Allen, J.; Haddox, R. Effect of the metal on electron transfer across self-assembled monolayers. J. Phys. Chem. B. 2001, 105, 3088-3092.

14. (a) Chidsey, C.E.D. Free energy and temperature dependence of electron transfer at the metalelectrolyte interface. Science 1991, 251, 919-922. (b) Chidsey, C.E.D.; Bertozzi, C.R.; Putvinski, T.M.; Mujsce, A.M. Coadsorption of ferrocene-terminated and unsubstituted alkanethiols on gold: electroactive self assembled monolayers. J. Am. Chem. Soc. 1990, 112, 4301-4306.

15. (a) Miller, C.; Cuendet, P.; Gratzel, M. Adsorbed $\omega$ - hydroxy thiol monolayers on gold electrodes: evidence for electron tunneling to redox species in solution. J. Phys. Chem. 1991, 95, 877-886. (b) Miller, C.; Gratzel, M. Electrochemistry at $\omega$-hydroxy thiol coated electrodes.2. Measurement of the density of electronic states distributions for several outer-sphere redox couples. J. Phys. Chem. 1991, 95, 5225-5233. (c) Becka, A.M.; Miller, C.J. Electrochemistry at $\omega$-hydroxy thiol coated electrodes. 3. Voltage independence of the electron tunneling barrier and measurements of redox kinetics at large overpotentials. J. Phys. Chem. 1992, 96, 2657-2668. 
16. (a) Forster, R.J.; Faulkner, L.R. Electrochemistry of spontaneously adsorbed monolayers. Equilibrium properties and fundamental electron transfer characteristics. J. Am. Chem. Soc. 1994, 116, 5444-5452. (b) Forster, R.J.; Faulkner, L.R. Electrochemistry of spontaneously adsorbed monolayers. Effects of solvent, potential and temperature on electron transfer dynamics. J. Am. Chem. Soc. 1994, 116, 5453-5461. (c) Forster, R.J.; O'Kelly, J.P. pH modulated heterogeneous electron transfer across metal/monolayer interfaces. J. Phys. Chem. 1996, 100, 3695-3704. (d) Forster, R.J.; Keyes, T.E. Tetrazine bridged osmium dimmers: electrochemical vs photoinduced electron transfer. J. Phys. Chem. B. 2001, 105, 8829-8837.

17. Finklea, H.O.; Hanshew, D.D. Electron transfer kinetics in organized thiol monolayers with attached pentaammine(pyridine)ruthenium redox centers. J. Am. Chem. Soc. 1992, 114, 3173-3181.

18. (a) Nahir, T.M.; Bowden, E.F. The distribution of standard rate constants for the electron transfer between thiol-modified gold electrodes and adsorbed cytochrome c. J. Electroanal. Chem. 1996, 410, 9-13. (b) Nahir, T.M.; Clark, R.A.; Bowden, E.F. Linear sweep voltammetry of irreversible electron transfer in surface confined species using the Marcus theory. Anal. Chem. 1994, 66, 25952598.

19. (a) Marcus, R.A. Symmetry or asymmetry of $\mathrm{k}_{\mathrm{ET}}$ and $\mathrm{i}_{\mathrm{STM}}$ vs. potential curves. J. Chem. Soc., Faraday Trans. 1996, 92, 3905-3908. (b) Gosavi, S.; Gao, Y.Q.; Marcus, R.A. Temperature dependence of the electronic factor in the nonadiabatic electron transfer at metal and semiconductor electrodes. J. Electroanal. Chem. 2001, 500, 71-77. (c) Gosavi, S.; Marcus, R.A. Nonadiabatic electron transfer at metal surfaces. J. Phys. Chem. B. 2000, 104, 2067-2072. (d) Hsu, C.P.; Application of the sequential formula: the electronic coupling and the distance dependence in the electron transfer of ferrocene-terminated alkanethiol monolayers. J. Electroanal. Chem. 1997, 438, 27-35. (e) Hsu, C.P.; Marcus, R.A. A sequential formula for electronic coupling in long range bridge assisted electron transfer: Formulation of theory and application to alkanethiol monolayers. J. Chem. Phys. 1997, 106, 584- 598 . (f) Ratner, M.A.; Bridge assisted electron transfer: effective electronic coupling. J. Phys. Chem. 1990, 94, 4877-4883.

20. (a) Xu, J.; Li, H-L.; Zhang, Y. Relationship between electronic tunneling coefficient and electrode potential investigated using self assembled alkanethiol monolayers on gold electrodes. J. Phys. Chem. 1993, 97, 11497-11500. (b) Arnold, S.; Feng, Z.Q.; Kakiuchi, T.; Knoll, W.; Niki, K. Investigation of the electrode reaction of cytochrome $\mathrm{c}$ through mixed self-assembled monolayers of alkanethiols on gold (111) surfaces. J. Electroanal. Chem. 1997, 438, 91-97. (c) Khoshtariya, D.E.; Dolidze, T.D.; Zusman, L.D.; Waldeck, D.H. Observation of the turnover between the solvent friction (overdamped) and tunneling (nonadiabatic) charge transfer mechanisms for a $\mathrm{Au} / \mathrm{Fe}(\mathrm{CN})_{6}{ }^{3 / 4-}$ electrode process and evidence for a freezing out of the Marcus barrier. J. Phys. Chem. A. 2001, 105, 1818-1829. (d) Yang, D.; Zi, M.; Chen, B.; Gao, Z. Separation of pinhole and tunneling electron transfer processes at self assembled polymeric monolayers on gold electrodes. $J$. Electroanal. Chem. 1999, 470, 114-119. (e) Kornyshev, A.A.; Kuznetsov, A.M.; Stimming, U.; Ulstrup, J. Rate processes in interfacial systems near continuous phase transitions. J. Phys. Chem. 1996, 100, 11184-11192.

21. (a) Creager, S.E.; Wooster, T.T. A new way of using ac voltammetry to study redox kinetics in electroactive monolayers. Anal. Chem. 1998, 70, 4257-4263. (b) O'Connor, S.D.; Olsen, G.T.; Creager, S.E. A Nernstian electron source model for the ac voltammetric response of a reversible 
surface redox reaction using large amplitude ac voltages. J. Electroanal. Chem. 1999, 466, 197202. (c) Sumner, J.J.; Creager, S.E. Redox kinetics in monolayers on electrodes: electron transfer is sluggish for ferrocene groups buried within the monolayer interior. J. Phys. Chem. B. 2001, 105, 8739-8745. (d) Creager, S.; Yu, C.J.; Bamdad, C.; O’Connor, S.; Maclean, T.; Lam, E.; Chong, Y.; Olsen, G.T.; Luo, J.; Gozin, M.; Kayyem, J.F. Electron transfer at electrodes through conjugated 'molecular wire' bridges. J. Am. Chem. Soc. 1999, 121, 1059-1064. (e) Sumner, J.J.; Creager, S.E. Topological effects in bridge mediated electron transfer between redox molecules and metal electrodes. J. Am. Chem. Soc. 2000, 122, 11914-11920. (f) Sumner, J.J.; Weber, K.S.; Hockett, L.A.; Creager, S.E. Long range heterogeneous electron transfer between ferrocene and gold mediated by $\omega$-alkane and $\omega$-alkyl-carboxamide bridges. J. Phys. Chem. B. 2000, 104, 7449-7454.

22. Brown, A.P.; Anson, F.C. Cyclic and differential pulse voltammetric behaviour of reactants confined to the electrode surface. Anal. Chem 1977, 49, 1589-1595.

23. Albery, W.J.; Boutelle, M.G.; Colby, P.J.; Hillman, R.A. The kinetics of electron transfer in the thionine coated electrode. J. Electroanal. Chem. 1982, 133, 135-145.

24. Laviron, E. A multilayer model for the study of space distributed redox modified electrodes. Part 3. Influence of the interactions between the electroactive centers in the first layer on the linear potential sweep voltammograms. J. Electroanal. Chem. 1981, 122, 37-44.

25. Daifuku, H.; Aoki, K.; Tokuda, K.; Matsuda, H. Electrode kinetics of surfactant polypyridine osmium and ruthenium complexes confined to tin oxide electrodes in a monomolecular layer by the Langmuir-Blodgett method. J. Electroanal. Chem. 1985, 183, 1-26.

26. (a) Matsuda, H.; Aoki, K.; Tokuda, K. Theory of electrode reactions of redox couples confined to electrode surfaces at monolayer levels. Part 1. Expression of the current-potential relationship for simple redox reactions. J. Electroanal. Chem. 1987, 217, 1-13. (b) Matsuda, H.; Aoki, K.; Tokuda, $\mathrm{K}$. Theory of electrode reactions of redox couples confined to electrode surfaces at monolayer levels. Part 2. Cyclic voltammetry and ac impedance measurements. J. Electroanal. Chem. 1987, $217,15-32$.

27. (a) Oldham, K.B.; Myland, J.C. Fundamentals of electrochemical science. Academic Press, New York, 1994. Chapter 11, pp.413-414. (b) Bard, A.J.; Faulkner, L.R. Electrochemical methods: fundamentals and applications, $2^{\text {nd }}$ edition, 2001, Wiley, New York. Chapter 5, pp.191-197.

28. Bard, A.J.; Faulkner, L.R. Electrochemical methods: fundamentals and applications, $2^{\text {nd }}$ edition, 2001, Wiley, New York. Chapter 5, pp.162-163.

29. Spanier, J.; Oldham, K.B. An atlas of functions. 1987, Hemisphere Publishing Corporation, Washington. Chapter 41. pp.395- 403.

Sample Availability: Available from the authors.

(C) 2002 by MDPI (http://www.mdpi.net). Reproduction is permitted for noncommercial purposes. 